\title{
COMPLETE REDUCIBILITY AND COMMUTING SUBGROUPS
}

\author{
MICHAEL BATE, BENJAMIN MARTIN, AND GERHARD RÖHRLE
}

\begin{abstract}
Let $G$ be a reductive linear algebraic group over an algebraically closed field of characteristic $p \geq 0$. We study J-P. Serre's notion of $G$-complete reducibility for subgroups of $G$. Specifically, for a subgroup $H$ and a normal subgroup $N$ of $H$, we look at the relationship between $G$-complete reducibility of $N$ and of $H$, and show that these properties are equivalent if $H / N$ is linearly reductive, generalizing a result of Serre. We also study the case when $H=M N$ with $M$ a $G$-completely reducible subgroup of $G$ which normalizes $N$. In our principal result we show that if $G$ is connected, $N$ and $M$ are connected commuting $G$-completely reducible subgroups of $G$, and $p$ is good for $G$, then $H=M N$ is also $G$ completely reducible.
\end{abstract}

\section{InTRODUCTION}

Let $G$ be a reductive algebraic group defined over an algebraically closed field of characteristic $p \geq 0$ and suppose $H$ is a subgroup of $G$. Following Serre [13], we say that $H$ is $G$-completely reducible (or simply $G$-cr) if whenever $H$ is contained in a parabolic subgroup $P$ of $G$, then $H$ is contained in a Levi subgroup of $P$ (in fact, we slightly extend Serre's definition to cover the case when $G$ is not connected, see Section 2 for precise details). The notion of $G$-complete reducibility was introduced by J-P. Serre as a way of generalizing the notion of complete reducibility (semisimplicity) from representation theory; indeed, when $G=\mathrm{GL}(V)$, a subgroup $H$ is $G$-completely reducible if and only if $V$ is a semisimple module for $H[13]$.

This paper builds on the following result [1, Thm. 3.10], see also [9, Thm. 2]:

Theorem 1.1. Let $H$ be a closed subgroup of $G$ with closed normal subgroup $N$. If $H$ is $G$-completely reducible, then so is $N$.

In the case $G=\mathrm{GL}(V)$, this statement reduces to Clifford's Theorem from representation theory. Theorem 1.1 answers a question raised by J-P. Serre [13, p. 24], who also provides a partial converse [13, Property 5] under some restrictions on the quotient $H / N$; other partial converses are provided by [1, Thm. 3.14, Cor. 3.16], for example. Note the converse is not true in full generality: e.g., take $N=\{1\}$ and $H$ a non-trivial unipotent subgroup of $G^{0}$ (see Remark 2.3).

In this paper we investigate partial converses to Theorem 1.1 under various restrictions. In Section 3 we show that if the quotient group $H / N$ is linearly reductive, then $H$ is $G$ completely reducible if and only if $N$ is $G$-completely reducible (see Corollary [3.7); when $H / N$ is finite, our result gives Serre's converse as a special case. We consider the following question.

Key words and phrases. G-complete reducibility, commuting subgroups, Clifford's Theorem.

2000 Mathematics Subject Classification. 20G15, 14L24. 
Question 1.2. Let $H, N$ be subgroups of $G$ with $N$ normal in $H$. Let $M$ be any subgroup of $H$ such that $M N=H$. Is it true that $H$ is $G$-completely reducible if and only if $M$ and $N$ are $G$-completely reducible?

If $H$ is $G$-cr and $M$ is also normal in $H$, then $M$ and $N$ are $G$-cr, by Theorem 1.1. When $M$ is not assumed to be normal in $H$, it is easy to construct examples where $H$ is $G$-cr but $M$ is not: we can just take $G=H=N$ and $M$ to be a non- $G$-cr subgroup of $G$. There are examples even when $M$ is a complement to $N$ in $H$ (Examples 5.7 and [5.8); the problem here is that $N$ can fail to normalize $M$.

Now consider the other implication of Question 1.2. Unfortunately, even in the best possible case when $M$ and $N$ are connected disjoint commuting subgroups of $G$, so that $M$ is a complement to $N$ and $M$ is normal in $H$, the answer is no (see Example 5.3). However, this is a low characteristic phenomenon, as we show in our main result:

Theorem 1.3. Suppose that $G$ is connected and that $p$ is good for $G$ or $p>3$. Let $A$ and $B$ be commuting connected $G$-completely reducible subgroups of $G$. Then $A B$ is $G$-completely reducible.

Theorem [1.3 was first proved in [10, Prop. 40] under the assumption that $p>2 h-2$, where $h$ is the Coxeter number of $G$; this bound stems from Serre's result [14, Cor. 5.5] used in the proof of [10, Prop. 40]. It follows from Theorem 1.3 that [10, Thm. 2] in fact holds for $p$ good.

This paper is organized as follows. In Section 2 we recall some background material, mostly taken from [1] and [7]. In Section 3 we prove some results on $G$-complete reducibility relevant to Theorem 1.1. Section 4 contains the proof of Theorem [1.3. Here we rely heavily on the exhaustive work of Liebeck and Seitz [7, which is based on intricate case-by-case arguments; we blend further case-by-case arguments with the general results from the previous sections. In Section 5 we consider some counterexamples to the statement of Theorem 1.3 with the assumption of connectedness or the hypothesis on the characteristic removed.

\section{Notation And Preliminaries}

2.1. Basic Notation. Throughout, we work over an algebraically closed field $k$ of characteristic $p \geq 0$; we let $k^{*}$ denote the multiplicative group of $k$. By a subgroup of an algebraic group we mean a closed subgroup and by a homomorphism of algebraic groups we mean a group homomorphism which is also a morphism of algebraic varieties. Let $H$ be a linear algebraic group. We denote by $\overline{\langle S\rangle}$ the algebraic subgroup of $H$ generated by a subset $S$ of $H$. We let $Z(H)$ denote the centre of $H$ and $H^{0}$ the connected component of $H$ that contains 1 . If $K$ is a subgroup of $H$, then $C_{H}(K)$ is the centralizer of $K$ in $H$ and $N_{H}(K)$ is the normalizer of $K$ in $H$. For elements $a$ and $b$ of $H$, we denote the commutator $a b a^{-1} b^{-1}$ by $[a, b]$; similarly, for subgroups $A$ and $B$ of $H$, we denote the commutator subgroup $\overline{\langle[a, b] \mid a \in A, b \in B\rangle}$ by $[A, B]$.

If $f: H_{1} \rightarrow H_{2}$ is a homomorphism of algebraic groups, then we say $f$ is non-degenerate if $(\operatorname{Ker} f)^{0}$ is a torus; in particular, an isogeny (an epimorphism with finite kernel) is nondegenerate. We denote the rank of $H$ by rk $H$. For the set of cocharacters (one-parameter subgroups) of $H$ we write $Y(H)$; the elements of $Y(H)$ are the homomorphisms from $k^{*}$ to $H$. 
The unipotent radical of $H$ is denoted $R_{u}(H)$; it is the maximal connected normal unipotent subgroup of $H$. The algebraic group $H$ is called reductive if $R_{u}(H)=\{1\}$; note that we do not insist that a reductive group is connected. In particular, $H$ is reductive if it is simple as an algebraic group ( $H$ is said to be simple if $H$ is connected and the only proper normal subgroups of $H$ are finite). For a connected reductive group $H$, we can write $[H, H]=H_{1} \cdots H_{t}$, where the $H_{i}$ are commuting simple subgroups of $H$; we call these subgroups the simple factors of $H$. The algebraic group $H$ is called linearly reductive if all rational representations of $H$ are semisimple. It is known that if $p=0$, then $H$ is linearly reductive if and only if $H$ is reductive; in contrast, M. Nagata has shown ([11, §4, Thm. 2]) that if $p>0$, then $H$ is linearly reductive if and only if $H^{0}$ is a torus and $H / H^{0}$ has order coprime to $p$.

Throughout the paper $G$ denotes a reductive algebraic group, possibly non-connected. A subgroup of $G$ normalized by some maximal torus $T$ of $G$ is called a regular subgroup of $G$ (connected semisimple regular subgroups of connected reductive groups are often also referred to as subsystem subgroups, e.g., see [7]).

Let $\Psi=\Psi(G, T)$ denote the set of roots of $G$ with respect to a maximal torus $T$. Fix a Borel subgroup $B$ of $G$ containing $T$ and let $\Sigma=\Sigma(G, T)$ be the set of simple roots of $\Psi$ defined by $B$. Then $\Psi^{+}=\Psi(B, T)$ is the set of positive roots of $G$. For $\beta \in \Psi^{+}$write $\beta=\sum_{\alpha \in \Sigma} c_{\alpha \beta} \alpha$ with $c_{\alpha \beta} \in \mathbb{N}_{0}$. A prime $p$ is said to be good for $G$ if it does not divide any non-zero $c_{\alpha \beta}$, and $b a d$ otherwise. A prime $p$ is good for $G$ if and only if it is good for every simple factor of $G$ [15]; the bad primes for the simple groups are 2 for all groups except type $A_{n}, 3$ for the exceptional groups and 5 for type $E_{8}$.

Suppose $G$ acts on a variety $V$ and let $v \in V$. Then for each cocharacter $\lambda \in Y(G)$, we can define a morphism of varieties $\phi_{v, \lambda}: k^{*} \rightarrow V$ via the formula $\phi_{v, \lambda}(x)=\lambda(x) \cdot v$. If this morphism extends to a morphism $\bar{\phi}_{v, \lambda}: k \rightarrow V$, then we say that $\lim _{x \rightarrow 0} \lambda(x) \cdot v$ exists, and set this limit equal to $\bar{\phi}_{v, \lambda}(0)$; note that such an extension, if it exists, is necessarily unique. This procedure is especially important for us when $V=G$ and $G$ acts by conjugation.

2.2. G-Complete Reducibility. In [1, Sec. 6], Serre's original notion of G-complete reducibility is extended to include the case where $G$ is reductive but not necessarily connected (so that $G^{0}$ is a connected reductive group). The crucial ingredient of this extension is the introduction of so-called Richardson parabolic subgroups ( $R$-parabolic subgroups) of a reductive group $G$. We briefly recall the main definitions and results; for more details and further results, the reader is referred to [1, Sec. 6].

Definition 2.1. For each cocharacter $\lambda \in Y(G)$, let $P_{\lambda}=\left\{g \in G \mid \lim _{x \rightarrow 0} \lambda(x) g \lambda(x)^{-1}\right.$ exists $\}$. Recall that a subgroup $P$ of $G$ is parabolic if $G / P$ is a complete variety. The subgroup $P_{\lambda}$ is parabolic in this sense, but the converse is not true: e.g., if $G$ is finite, then every subgroup is parabolic, but the only subgroup of $G$ of the form $P_{\lambda}$ is $G$ itself. If we define $L_{\lambda}=\left\{g \in G \mid \lim _{x \rightarrow 0} \lambda(x) g \lambda(x)^{-1}=g\right\}$, then $P_{\lambda}=L_{\lambda} \ltimes R_{u}\left(P_{\lambda}\right)$, and we also have $R_{u}\left(P_{\lambda}\right)=$ $\left\{g \in G \mid \lim _{x \rightarrow 0} \lambda(x) g \lambda(x)^{-1}=1\right\}$. The subgroups $P_{\lambda}$ for $\lambda \in Y(G)$ are called the $R$-parabolic subgroups of $G$. Given an R-parabolic subgroup $P$, an $R$-Levi subgroup of $P$ is any subgroup $L_{\lambda}$ such that $\lambda \in Y(G)$ and $P=P_{\lambda}$. If $G$ is connected, then the R-parabolic subgroups (resp. R-Levi subgroups of R-parabolic subgroups) of $G$ are exactly the parabolic subgroups (resp. Levi subgroups of parabolic subgroups) of $G$; indeed, most of the theory of parabolic 
subgroups and Levi subgroups of connected reductive groups carries over to R-parabolic and R-Levi subgroups of arbitrary reductive groups. In particular, all R-Levi subgroups of an R-parabolic subgroup $P$ are conjugate under the action of $R_{u}(P)$.

We are often interested in reductive subgroups of reductive groups. If $H$ is a subgroup of $G$, then there is an obvious inclusion $Y(H) \subseteq Y(G)$ of the sets of cocharacters. When $H$ is reductive and $\lambda \in Y(H)$, there is then an R-parabolic subgroup of $H$ associated to $\lambda$, as well as an R-parabolic subgroup of $G$. In order to distinguish between R-parabolic subgroups associated to different subgroups of $G$, we use the notation $P_{\lambda}(H), L_{\lambda}(H)$, etc. where necessary, but we write $P_{\lambda}$ for $P_{\lambda}(G)$ and $L_{\lambda}$ for $L_{\lambda}(G)$. Note that $P_{\lambda}(H)=P_{\lambda} \cap H$, $L_{\lambda}(H)=L_{\lambda} \cap H$ and $R_{u}\left(P_{\lambda}(H)\right)=R_{u}\left(P_{\lambda}\right) \cap H$.

We now have the machinery in place to define exactly what we mean by a $G$-completely reducible subgroup in this more general setting; note that, by the remarks above, the following definition coincides with Serre's notion for connected $G$.

Definition 2.2. Suppose $H$ is a subgroup of $G$. We say $H$ is $G$-completely reducible ( $G$-cr for short) if whenever $H$ is contained in an R-parabolic subgroup of $G$, then there exists an R-Levi subgroup $L$ of $P$ with $H \subseteq L$. Equivalently, $H$ is $G$-completely reducible if whenever $H \subseteq P_{\lambda}$ for some $\lambda \in Y(G)$, there exists $\mu \in Y(G)$ such that $P_{\lambda}=P_{\mu}$ and $H \subseteq L_{\mu}$. Since all R-Levi subgroups of an R-parabolic subgroup $P$ are $R_{u}(P)$-conjugate, we have another formulation: $H$ is $G$-completely reducible if whenever $H \subseteq P_{\lambda}$ for some $\lambda \in Y(G)$, there exists $u \in R_{u}\left(P_{\lambda}\right)$ such that $H \subseteq u L_{\lambda} u^{-1}$.

Note that, for $G=\mathrm{GL}(V)$, a subgroup $H$ is $G$-completely reducible if and only if $V$ is a semisimple $H$-module.

Remark 2.3. If $G$ is connected, then any $G$-completely reducible subgroup $H$ is reductive: in fact $H$ cannot normalize any non-trivial unipotent subgroup of $G^{0}$. This was shown by Serre in [14, §4.1]. Serre's argument gives the same result for non-connected $G$, cf. [8, Prop. $5.4(\mathrm{~b})]$.

Sometimes we come across subgroups of $G$ which are not contained in any R-parabolic subgroup of $G$; these subgroups are trivially $G$-completely reducible. Following Serre again, we call these subgroups $G$-irreducible $(G$-ir). Note that, for $G=\operatorname{GL}(V)$, a subgroup $H$ is $G$-irreducible if and only if $V$ is an irreducible $H$-module.

We recall some results on $G$-complete reducibility (mainly from [1]) which we require in the sequel. The reader should note that many of the results in [1] were proved first for connected groups and then generalized to the non-connected case; we give separate references where appropriate from [1] for the connected and non-connected versions of each result (see the paragraph immediately preceding [1, Sec. 6.2]).

Our first result (see [5, Lem. 11.24] for a proof), together with Remark 2.3, shows that in characteristic zero, a subgroup of $G$ is $G$-completely reducible if and only if it is reductive; thus our results only have independent interest in positive characteristic. This equivalence is not true in positive characteristic. For we can take a reductive but not linearly reductive group $H$ and map it into some $\mathrm{GL}(V)$ in a non-semisimple way; the image of $H$ is then reductive but not $\mathrm{GL}(V)$-cr.

Lemma 2.4. If $H$ is a linearly reductive subgroup of $G$, then $H$ is $G$-completely reducible. 
Regular subgroups play an important rôle in many of our proofs; the following result [1, Prop. 3.20] shows that these subgroups are $G$-completely reducible.

Lemma 2.5. If $H$ is a regular reductive subgroup of $G$, then $H$ is $G$-completely reducible.

We need a useful preliminary result.

Lemma 2.6. Let $N$ be a normal subgroup of $G$. Then there exists a subgroup $M$ of $G$ such that $M N=G, M \cap N$ is a finite normal subgroup of $M, M^{0} \cap N^{0}$ is central in both $M^{0}$ and $N^{0}$, and $M^{0}$ commutes with $N$.

Proof. The existence of $M$ and all of its properties except the last follow from [1, Lem. 6.14] and its proof. To show that $M^{0}$ commutes with $N$, we observe that for any $x \in N$, the connected set $\left\{[x, y] \mid y \in M^{0}\right\}$ is contained in the finite set $M \cap N$ and hence must be trivial.

The next result (see [1, Lem. 2.12, Sec. 6.2]) shows that the concept of complete reducibility behaves well with respect to certain homomorphisms. Observe that Lemma 2.7(ii)(b) applies in particular to isogenies; we use this special case frequently in the sequel.

Lemma 2.7. Let $G_{1}$ and $G_{2}$ be reductive groups.

(i) Let $H$ be a subgroup of $G_{1} \times G_{2}$. Let $\pi_{i}: G_{1} \times G_{2} \rightarrow G_{i}$ be the canonical projection for $i=1,2$. Then $H$ is $\left(G_{1} \times G_{2}\right)$-completely reducible if and only if $\pi_{i}(H)$ is $G_{i^{-}}$ completely reducible for $i=1,2$.

(ii) Let $f: G_{1} \rightarrow G_{2}$ be an epimorphism. Let $H_{1}$ (resp. $H_{2}$ ) be a subgroup of $G_{1}$ (resp. $\left.G_{2}\right)$.

(a) If $H_{1}$ is $G_{1}$-completely reducible, then $f\left(H_{1}\right)$ is $G_{2}$-completely reducible.

(b) If $f$ is non-degenerate, then $H_{1}$ is $G_{1}$-completely reducible if and only if $f\left(H_{1}\right)$ is $\mathrm{G}_{2}$-completely reducible, and $\mathrm{H}_{2}$ is $G_{2}$-completely reducible if and only if $f^{-1}\left(\mathrm{H}_{2}\right)$ is $G_{1}$-completely reducible.

In particular, let $G=G_{1} \times G_{2}$ and consider the normal subgroup $G_{1}$ of $G$. If $H$ is a subgroup of $G_{1}$, then $H$ is $G$-completely reducible if and only if $H$ is $G_{1}$-completely reducible. We extend this result to cover the case of a normal subgroup which is not necessarily a direct factor of $G$.

Proposition 2.8. Let $K \subseteq N \subseteq G$ be subgroups of $G$ with $N$ normal in $G$. Then $K$ is $G$-completely reducible if and only if $K$ is $N$-completely reducible.

Proof. Let $M$ be as in Lemma 2.6. Suppose $K$ is $G$-cr. Let $\lambda \in Y(N)$ such that $K \subseteq P_{\lambda}(N)$. As $K$ is $G$-cr, there exists $u \in R_{u}\left(P_{\lambda}\right)$ such that $K \subseteq u L_{\lambda} u^{-1}$. Now $R_{u}\left(P_{\lambda}\right) \subseteq G^{0}$, so we can write $u=u_{1} u_{2}$ with $u_{1} \in N^{0}$ and $u_{2} \in M^{0}$. Since $\lambda$ centralizes $M^{0}$, we see that $u_{1}^{\prime}:=\lim _{x \rightarrow 0} \lambda(x) u_{1} \lambda(x)^{-1}$ must exist and equal $u_{2}^{-1}$. Then $u_{2} \in M^{0} \cap N^{0}$, which consists of semisimple elements, so $u_{2}=1=u_{1}^{\prime}$ and $u_{1} \in R_{u}\left(P_{\lambda}(N)\right)$. Thus $K \subseteq u_{1} L_{\lambda}(N) u_{1}^{-1}$, an R-Levi subgroup of $P_{\lambda}(N)$. This shows that $K$ is $N$-cr.

Conversely, suppose $K$ is $N$-cr. Let $\lambda \in Y(G)$ such that $K \subseteq P_{\lambda}$. Since $M^{0}$ commutes with $N$, we can write $\lambda=\sigma+\tau$, where $\sigma \in Y(M)$ and $\tau \in Y(N)$. Since $\sigma$ centralizes $N$, $\sigma$ centralizes $K$, so $K \subseteq P_{\tau} \cap N=P_{\tau}(N)$. As $K$ is $N$-cr, there exists $u \in R_{u}\left(P_{\tau}(N)\right)=$ $R_{u}\left(P_{\tau}\right) \cap N=R_{u}\left(P_{\lambda}\right) \cap N$ such that $u K u^{-1} \subseteq L_{\tau}(N)=L_{\lambda} \cap N$. Thus $K$ is $G$-cr, as required. 
The following three results allow us to relate $G$-complete reducibility and $H$-complete reducibility for subgroups of some important subgroups $H$ of $G$. The first result ([1, Cor. 3.21]) makes sense because, if $S$ is a linearly reductive group acting on $G$ by automorphisms, then $C_{G}(S)$ is reductive [12, Prop. 10.1.5].

Proposition 2.9. Let $S$ be a linearly reductive group acting on $G$ by automorphisms and let $H=C_{G}(S)^{0}$. Suppose $K$ is a subgroup of $H$. Then $K$ is $H$-completely reducible if and only if $K$ is $G$-completely reducible.

This is of particular use to us when $G$ is simple and $S$ is the finite group generated by a graph automorphism of $G$. The next result is a corollary of Proposition 2.9 when $G$ is connected; the point is that any R-Levi subgroup of an R-parabolic subgroup of $G$ is the centralizer of a torus. The extension to non-connected groups is not hard (see [1, Cor. 3.22, Sec. 6.3]).

Corollary 2.10. Let $L$ be an $R$-Levi subgroup of some $R$-parabolic subgroup of $G$. Then a subgroup $K$ of $L$ is $G$-completely reducible if and only if it is L-completely reducible.

The next result is [1, Thm. 3.26] when $G$ is connected. The proof given there still applies for non-connected $G$ by Proposition 2.12 below, since $H$ is assumed to be connected.

Proposition 2.11. Suppose that $p$ is good for $G^{0}$. Let $H$ be a regular connected reductive subgroup of $G$ and suppose $K$ is a subgroup of $H$. Then $K$ is $H$-completely reducible if and only if $K$ is $G$-completely reducible.

The following result is a generalization of [1, Lem. 6.12(i)]. Note that a finite-index subgroup of a reductive group is reductive.

Proposition 2.12. Let $K \subseteq H$ be subgroups of $G$, with $H$ of finite index in $G$. Then $K$ is $H$-completely reducible if and only if $K$ is $G$-completely reducible.

Proof. Suppose $K$ is $G$-cr. Let $P$ be an R-parabolic subgroup of $H$ with $K \subseteq P$. We can write $P=P_{\lambda}(H)$ for some $\lambda \in Y(H)$. Then $K \subseteq P_{\lambda}$. Since $K$ is $G$-cr, there exists $u \in R_{u}\left(P_{\lambda}\right)$ such that $u K u^{-1} \subseteq L_{\lambda}$. We have $R_{u}\left(P_{\lambda}(H)\right)=H \cap R_{u}\left(P_{\lambda}\right)=H^{0} \cap R_{u}\left(P_{\lambda}\right)=$ $G^{0} \cap R_{u}\left(P_{\lambda}\right)=R_{u}\left(P_{\lambda}\right)$, so $u$ belongs to $R_{u}\left(P_{\lambda}(H)\right)$ and $u K u^{-1} \subseteq H \cap L_{\lambda}=L_{\lambda}(H)$. Thus $K$ is $H$-cr. The argument in the other direction is similar.

We obtain a corollary which strengthens Proposition 2.8.

Corollary 2.13. Let $N$ be a subgroup of $G$ such that the number of $G$-conjugates of $N$ is finite, and let $K$ be a subgroup of $N$. Then $K$ is $G$-completely reducible if and only if $K$ is $N$-completely reducible.

Proof. The subgroup $G_{1}:=N_{G}(N)$ has finite index in $G$. Then $G_{1}$ is reductive, so its normal subgroup $N$ is also reductive. The result now follows from Propositions 2.8 and 2.12 .

Remark 2.14. Let $K, H$ be subgroups of $G$ with $H$ of finite index in $G$. It need not be true that if $K$ is $G$-completely reducible then $K \cap H$ is $H$-completely reducible. For example, let $p=2$, let $\phi$ be an irreducible embedding of the symmetric group $S_{3}$ in $\mathrm{SL}_{2}$, let $G=S_{3} \times \mathrm{SL}_{2}$ and let $K=\left\{g \phi(g) \mid g \in S_{3}\right\}$. Let $C_{2}$ be a cyclic subgroup of $S_{3}$ of order 2 and let $H=C_{2} \times \mathrm{SL}_{2} \subseteq G$. It is easily checked that $K$ is $G$-ir but $K \cap H$ is not $H$-cr. 
Some of the proofs in Section 4 rely on the monograph [7] of M. Liebeck and G. Seitz. We recall some notation and results from this paper. For the rest of this section, assume $G$ is simple (hence connected) and of exceptional type. For convenience, we take $p$ to be $\infty$ rather than zero if $k$ has characteristic zero. For certain simple subgroups $X$ of $G$, Liebeck and Seitz provide a positive integer $N(X, G)$ given in the table at the top of page 2 of [7]. We reproduce this table for ease of reference (Table 1).

\begin{tabular}{r|rrrrr} 
& $G=E_{8}$ & $E_{7}$ & $E_{6}$ & $F_{4}$ & $G_{2}$ \\
\hline$X=A_{1}$ & 7 & 7 & 5 & 3 & 3 \\
$A_{2}$ & 5 & 5 & 3 & 3 & \\
$B_{2}$ & 5 & 3 & 3 & 2 & \\
$G_{2}$ & 7 & 7 & 3 & 2 & \\
$A_{3}$ & 2 & 2 & 2 & & \\
$B_{3}$ & 2 & 2 & 2 & 2 & \\
$C_{3}$ & 3 & 2 & 2 & 2 & \\
$B_{4}, C_{4}, D_{4}$ & 2 & 2 & 2 & &
\end{tabular}

TABLE 1. The bounds $N(X, G)$ from [7].

For example, if $X$ has type $A_{3}$ and $G$ has type $E_{6}$, then $N(X, G)=2$. If the pair $(X, G)$ is not in the table, then set $N(X, G)=1$. More generally, if $X$ is a connected reductive subgroup of $G$, and $X_{1}, \ldots, X_{t}$ are the simple factors of $X$, then define

$$
N(X, G)=\max \left(N\left(X_{i}, G\right) \mid 1 \leq i \leq t\right),
$$

where we take $N(X, G)=1$ if $X$ is a torus. With this definition, we can restate [7, Thm. 1, Thm. 3.8] in our language.

Theorem 2.15. Let $X$ be a connected reductive subgroup of $G$ and assume that $p>N(X, G)$. Then $X$ is $G$-completely reducible.

Theorem 2.16. Let $C$ be a simple subgroup of $G$ such that $C$ is of classical type. Suppose that $X$ is a connected reductive subgroup of $C$ and that $p>N(X, G)$. When $(X, p)=\left(B_{l}, 2\right)$ or $\left(C_{l}, 2\right)$, assume that $C \neq B_{r}$ or $C_{r}$. Then $X$ is $C$-completely reducible.

Note that whether a given $X$ is $G$-completely reducible depends only on the Dynkin type of $G$ and not on its isogeny class (Lemma 2.7(ii)(b)), so there is no harm in labelling $G$ only by its Dynkin type.

Remark 2.17. Tables 8.1-8.5 of [7] give the connected simple subgroups $X$ of an exceptional group $G$, the connected centralizers $C_{G}(X)^{0}$, and the minimal connected semisimple regular subgroups of $G$ that contain $X$, under the hypothesis that $p>N(X, G)$. Note that these tables give $X$ up to $\operatorname{Aut}(G)$-conjugacy. We give an example to point out one consequence of this. Suppose $p>2$. A group of type $D_{6}$ contains a subgroup of type $B_{5}$ as the connected centralizer of a graph automorphism of order 2. A group of type $E_{7}$ contains a group of type $D_{6}$ as a Levi subgroup. From Table 8.2 of [7], we see that there is exactly one copy up to $\operatorname{Aut}(G)$-conjugacy of a group $X$ of type $B_{5}$ in a group $G$ of type $E_{7}$, so it must be the subgroup we already know: that is, it must be the connected centralizer of a graph automorphism of order 2 of the $D_{6}$-subgroup of $E_{7}$. We use arguments of this kind repeatedly in the proof of Theorem 1.3 . 


\section{Converses to Theorem 1.1}

In this section we give several results providing conditions under which the converse of Theorem 1.1 holds, culminating in Corollary 3.7, which generalizes a result of Serre. We also prove some technical results which prepare the ground for the proof of our main result in Section 4. We begin with a refinement of [1, Prop. 3.19].

Theorem 3.1. Suppose $K \subseteq H \subseteq M$ are subgroups of $G$ such that $M$ is reductive.

(a) Suppose $M$ contains a maximal torus of $C_{G}(K)$.

(i) If $H$ is $G$-completely reducible, then $M$ is $G$-completely reducible.

(ii) If $K$ is $G$-completely reducible, then $M$ is $G$-completely reducible.

(b) Suppose $C_{G}(K)^{0} \subseteq M$.

(i) If $H$ is $G$-completely reducible, then $H$ is $M$-completely reducible.

(ii) If $K$ is $G$-completely reducible and $H$ is $M$-completely reducible, then $H$ is $G$ completely reducible.

Proof. (a). Note that part (ii) follows from part (i), just taking $H$ to be $K$. To prove part (i), suppose $P$ is an R-parabolic subgroup of $G$ containing $M$. Then $H$ lies in an R-Levi subgroup $L$ of $P$, since $H$ is $G$-cr, so $K$ lies in $L$. Let $S$ be a maximal torus of $C_{G}(K)$ such that $S \subseteq M$. Then $S$ is a maximal torus of $C_{P}(K)$, so, after conjugating $L$ by some element of $C_{P}(K)$, we can assume that $Z(L)^{0} \subseteq S$. Pick $\lambda \in Y(G)$ such that $P=P_{\lambda}$ and $L=L_{\lambda}$. Then $\lambda \in Y(S) \subseteq Y(M)$. We have $P_{\lambda}(M)=P_{\lambda} \cap M=M$, so $\lambda \in Y(Z(M))$, by [1, Lem. 2.4]. Thus $M \subseteq L_{\lambda}=L$. This shows that $M$ is $G$-cr.

(b)(i). We have $C_{G}(H)^{0} \subseteq C_{G}(K)^{0} \subseteq M$ by hypothesis. Now [1, Prop. 3.19] implies that if $H$ is $G$-cr, then $H$ is $M$-cr.

(ii). Suppose $P$ is an R-parabolic subgroup of $G$ containing $H$; then $K \subseteq P$ also. We are given that $K$ is $G$-cr, so there exists an R-Levi subgroup $L$ of $P$ with $K \subseteq L$. Let $\lambda \in Y(G)$ be such that $P=P_{\lambda}$ and $L=L_{\lambda}$. Then, since $K \subseteq L=C_{G}\left(\lambda\left(k^{*}\right)\right)$, we have $\lambda \in Y\left(C_{G}(K)^{0}\right) \subseteq Y(M)$. Thus $P_{\lambda}(M)$ is an R-parabolic subgroup of $M$ containing $H$. Since $H$ is $M$-cr, there exists $u \in R_{u}\left(P_{\lambda}(M)\right)=R_{u}\left(P_{\lambda}\right) \cap M$ such that $H \subseteq u L_{\lambda}(M) u^{-1}$. Hence $H \subseteq u L_{\lambda} u^{-1}$, an R-Levi subgroup of $P$. This shows that $H$ is $G$-cr.

Remark 3.2. (a) Note that in part (a) of Theorem 3.1 we only require $M$ to contain a maximal torus of $C_{G}(K)$, rather than all of $C_{G}(K)^{0}$. Part (b) fails under this weaker hypothesis, however. For example, we can take $K$ to be $\{1\}$ and $H$ to be a subgroup of $M$, where $M$ contains a maximal torus of $G$. There exist examples in which $H$ is $M$-cr but not $G$-cr, and others in which $H$ is $G$-cr but not $M$-cr. For the former, see [1, Ex. 3.45]: we take $H$ to be $\mathrm{Sp}_{m}$ embedded diagonally in $M:=\mathrm{Sp}_{m} \times \mathrm{Sp}_{m}$ inside $G:=\mathrm{Sp}_{2 m}$, where $m \geq 4$ is even. For the latter, we can take $H$ and $M$ to be certain subgroups of a simple group of type $G_{2}$ in characteristic 2; see [2, Prop. 7.17].

(b) Putting $M=H$ in Theorem 3.1, we obtain the following result: if $K$ is a $G$-cr subgroup of $G$, then any reductive subgroup $H$ of $G$ containing $K C_{G}(K)^{0}$ is also $G$-cr (note that $H$ is $H$-cr). This is a strengthening of [1, Thm. 3.14].

Corollary 3.3. Suppose $N \subseteq H$ are subgroups of $G, N$ is normal in $H$ and $N$ is $G$ completely reducible. Then $H$ is $G$-completely reducible if and only if $H$ is $N_{G}(N)$-completely reducible. 
Proof. This follows from Theorem 3.1(b), setting $K=N$ and $M=N_{G}(N)$. Note that since $N$ is $G$-cr, $N_{G}(N)$ is reductive, by the non-connected version of [1, Prop. 3.12].

Theorem 3.4. Suppose $N \subseteq H$ are subgroups of $G$ and $N$ is normal in $G$. Then $H$ is $G$-completely reducible if and only if $H / N$ is $G / N$-completely reducible.

Proof. Let $\pi: G \rightarrow G / N$ denote the canonical homomorphism. We first perform a series of reductions, using results from [1. Let $M$ be as in Lemma 2.6. Since $N$ is normal in $G, Z\left(N^{0}\right)^{0}$ is normal in $G$; the canonical map $G \rightarrow G / Z\left(N^{0}\right)^{0}$ is non-degenerate, so by Lemma 2.7(ii)(b) we may assume that $Z\left(N^{0}\right)^{0}=\{1\}$. The map $M \ltimes N \rightarrow G$ induced by multiplication is also non-degenerate, so we may assume that $G=M \ltimes N$ and, therefore, that the map $\pi: G \rightarrow G / N$ is the projection onto the first factor.

Now suppose $\lambda \in Y(M)$. Then, since $N$ and $M^{0}$ commute, $\lambda\left(k^{*}\right)$ centralizes $N$. By [1, Lem. 6.15(i),(ii)], we have

$$
\begin{array}{rlrl}
\pi\left(P_{\lambda}\right) & =P_{\lambda}(M), & & \pi^{-1}\left(P_{\lambda}(M)\right)=P_{\lambda}, \\
\pi\left(L_{\lambda}\right)=L_{\lambda}(M), & & \pi^{-1}\left(L_{\lambda}(M)\right)=L_{\lambda} .
\end{array}
$$

If $P=P_{\mu}$ is an R-parabolic subgroup of $G$ containing $H$, then we can write $\mu=\lambda+\nu$, where $\lambda \in Y(M)$ and $\nu \in Y(N)$. Since $P$ contains $H$, it contains $N$; but $N$ is normal in $G$, and hence $N$ is $G$-cr by Theorem 1.1, which means that $N$ is contained in some R-Levi subgroup of $P$. Moreover, normality of $N$ in $P$ implies $N$ is contained in every R-Levi subgroup of $P$; in particular, $N \subseteq L_{\mu}$, so $\mu\left(k^{*}\right)$ centralizes $N$. We have already noted that $\lambda\left(k^{*}\right)$ centralizes $N$ for any $\lambda \in Y(M)$. Thus $\nu\left(k^{*}\right)$ centralizes $N$, and, since $Z\left(N^{0}\right)^{0}=\{1\}, \nu$ is trivial. We can finally conclude that $\mu \in Y(M)$.

It is now easy to see from Equations (3.5) and (3.6) that $H$ is contained in an R-parabolic (resp. R-Levi) subgroup of $G$ if and only if $H / N$ is contained in the corresponding Rparabolic (resp. R-Levi) subgroup of $G / N$. Thus $H$ is $G$-cr if and only if $H / N$ is $G / N$-cr, as required.

We can now prove the main result of this section.

Corollary 3.7. Suppose that $N \subseteq H$ are subgroups of $G$ with $N$ normal in $H$.

(i) If $N$ is $G$-completely reducible, then $H$ is $G$-completely reducible if and only if $H / N$ is $N_{G}(N) / N$-completely reducible.

(ii) If $H / N$ is linearly reductive, then $H$ is $G$-completely reducible if and only if $N$ is $G$-completely reducible.

Proof. (i). By Corollary 3.3, $H$ is $G$-cr if and only if $H$ is $N_{G}(N)$-cr. By Theorem 3.4 applied to the inclusions $N \subseteq H \subseteq N_{G}(N)$, it follows that $H$ is $N_{G}(N)$-cr if and only if $H / N$ is $N_{G}(N) / N$-cr. This proves the result.

(ii). Suppose $H / N$ is linearly reductive. If $N$ is $G$-cr, then $N_{G}(N)$ is a reductive group and so is the quotient $N_{G}(N) / N$. Thus $H / N$ is a linearly reductive subgroup of $N_{G}(N) / N$. By Lemma 2.4, $H / N$ is $N_{G}(N) / N$-cr, and hence $H$ is $G$-cr, by part (i). On the other hand, if $H$ is $G$-cr, then $N$ is $G$-cr, by Theorem 1.1 .

Remark 3.8. Note that Serre's result [13, Property 5] is a special case of the reverse implication of Corollary 3.7(ii); the finite linearly reductive groups are exactly those whose orders are coprime to $p=$ char $k$. Corollary 3.7(ii) answers a question posed by B. Külshammer. 
Corollary 3.7(ii) provides a useful criterion to ensure that $G$-complete reducibility of $H$ and of $N$ are equivalent. However, there are many examples where $H$ and $N$ are $G$-completely reducible, but $H / N$ is not linearly reductive. The problem in general is that $G$-complete reducibility of $N$ and $H$ depends not only on how $N$ sits inside $H$, but also on how $H$ sits inside $G$. Therefore, to make more progress, one has to impose further conditions on $H$. In the next section we consider the case when $H=M N$, where $M$ commutes with $N$ and $M$ is also $G$-completely reducible. We now give some results applicable to this special case, the first of which is used many times in the proof of Theorem 1.3 .

Proposition 3.9. Suppose $N$ is a $G$-completely reducible subgroup of $G$ and $M$ is a subgroup of $C_{G}(N)$. Then $M$ is $C_{G}(N)$-completely reducible if and only if $M N$ is $G$-completely reducible.

Proof. By Corollaries 3.3 and 3.7(i), $M N$ is $G$-cr if and only if $M N$ is $N_{G}(N)$-cr if and only if $M N / N$ is $N_{G}(N) / N$-cr. Now $C_{G}(N) N / N$ is normal in $N_{G}(N) / N$, so $M N / N$ is $N_{G}(N) / N$-cr if and only if $M N / N$ is $C_{G}(N) N / N$-cr (Proposition 2.8). Let $f: C_{G}(N) \rightarrow C_{G}(N) N / N$ be the inclusion of $C_{G}(N)$ in $C_{G}(N) N$ followed by the canonical projection from $C_{G}(N) N$ to $C_{G}(N) N / N$. The connected kernel of $f$ is $\left(N \cap C_{G}(N)\right)^{0}=Z(N)^{0}$, which is a torus since $N$ is reductive, and $f(M)$ is $M N / N$. It follows from Proposition 2.7(ii) that $M$ is $C_{G}(N)$-cr if and only if $M N / N$ is $C_{G}(N) N / N$-cr, which proves the result.

Corollary 3.10. Suppose $N$ is $G$-completely reducible and $M$ is a subgroup of $C_{G}(N)$. If $M$ is $C_{G}(N)$-completely reducible, then $M$ is $G$-completely reducible.

Proof. Under these hypotheses, $M N$ is $G$-cr, by Proposition 3.9. But $M$ is a normal subgroup of $M N$, so $M$ is $G$-cr, by Theorem 1.1 .

In Section 5 we will show that the converse of Corollary 3.10 is not true in general, although it is true in the important special case of Theorem 1.3.

We finish this section with a result which sometimes allows us to reduce to the case of commuting subgroups.

Lemma 3.11. Let $A, B$ be subgroups of $G$ such that $A$ normalizes $B,[A, B]$ is linearly reductive and $[A, B]$ centralizes $A$. Then $A$ is $G$-completely reducible if and only if $C_{A}(B)$ is $G$-completely reducible, and $A B$ is $G$-completely reducible if and only if $C_{A}(B) B$ is $G$ completely reducible.

Proof. Note that $C_{A}(B), C_{A}(B) B$ are normal subgroups of $A, A B$ respectively, so by Corollary 3.7(ii), it is enough to show that $A / C_{A}(B)$ and $A B / C_{A}(B) B$ are linearly reductive. By a standard DCC argument, we can choose $b_{1}, \ldots, b_{r} \in B$ for some $r \in \mathbb{N}$ such that $C_{A}(B)=C_{A}\left(\left\langle b_{1}, \ldots, b_{r}\right\rangle\right)$. Define $\phi: A \rightarrow[A, B]^{r}$ by

$$
\phi(a)=\left(\left[a^{-1}, b_{1}\right], \ldots,\left[a^{-1}, b_{r}\right]\right) .
$$

Since $A$ commutes with $[A, B]$, we have

$$
\left[a^{-1}, b_{i}\right]\left[a^{\prime-1}, b_{i}\right]=a^{\prime-1}\left[a^{-1}, b_{i}\right] b_{i} a^{\prime} b_{i}^{-1}=\left(a a^{\prime}\right)^{-1} b_{i}\left(a a^{\prime}\right) b_{i}^{-1}=\left[\left(a a^{\prime}\right)^{-1}, b_{i}\right],
$$

for each $i$ and each $a, a^{\prime} \in A$. It follows that $\phi$ is a homomorphism from $A$ to the linearly reductive group $[A, B]^{r}$ with kernel $C_{A}(B)$, whence $A / C_{A}(B)$ is linearly reductive. The obvious map from $A / C_{A}(B)$ to $A B / C_{A}(B) B$ is surjective, so $A B / C_{A}(B) B$ is also linearly reductive, as required. 


\section{Proof of Theorem 1.3}

In this section we prove our main result, Theorem 1.3, via a series of reductions. It is immediate from Lemma 2.4 that Theorem 1.3 holds if $G$ is a torus. The next step is to reduce to the case when $G$ is simple. We begin with a technical definition.

Definition 4.1. Suppose $G$ is connected. Write $G=G_{1} \cdots G_{r} Z$, where the subgroups $G_{i}$ are the simple factors of $G$, and $Z$ is a central torus. Let $\tilde{G}=G_{1} \times \cdots \times G_{r} \times Z$, let $\phi$ be the isogeny from $\tilde{G}$ onto $G$ induced by multiplication, and let $\pi_{i}$ denote the projection of $\tilde{G}$ onto $G_{i}$ for each $i$. If $X$ is a connected reductive subgroup of $G$, we call the subgroup $\pi_{i}\left(\phi^{-1}(X)\right)$ of $G_{i}$ the projection of $X$ to the simple factor $G_{i}$, and we denote it by $X_{i}$.

Lemma 4.2. Keep the notation of Definition 4.1. Then $X$ is $G$-completely reducible if and only if $X_{i}$ is $G_{i}$-completely reducible for every $i$. If $X$ is connected, then $X$ is $G$-completely reducible if and only if $X_{i}^{0}$ is $G_{i}$-completely reducible for every $i$.

Proof. By Lemma 2.7(ii)(b), $X$ is $G$-cr if and only if $\phi^{-1}(X)$ is $\tilde{G}$-cr. Let $\pi$ denote the projection of $\tilde{G}$ onto the central torus $Z$. Since $Z$ is a torus, $\pi\left(\phi^{-1}(X)\right)$ is $Z$-cr (Lemma 2.4). Thus, by Lemma 2.7(i), $\phi^{-1}(X)$ is $\tilde{G}$-cr if and only if $X_{i}$ is $G_{i}$-cr for each $i$. This proves the first assertion. If $X$ is connected, then for each $i, X_{i}$ is generated by commuting subgroups $X_{i}^{0}$ and $\pi_{i}(\operatorname{ker}(\phi))$. Since $\pi_{i}(\operatorname{ker}(\phi))$ is linearly reductive, $X_{i}$ is $G_{i}$-cr if and only if $X_{i}^{0}$ is $G_{i}$-cr. For, if $X_{i}$ is $G$-cr, then $X_{i}^{0}$ is $G$-cr by Theorem 1.1. Conversely, if $X_{i}^{0}$ is $G$-cr, then $\pi_{i}(\operatorname{ker}(\phi))$, being linearly reductive, is $C_{G}\left(X_{i}^{0}\right)$-cr by Lemma 2.4 , so $X_{i}$ is $G$-cr by Proposition 3.9. The second assertion of the lemma now follows from the first.

Lemma 4.3. Let $f: G_{1} \rightarrow G_{2}$ be an isogeny of connected reductive groups. Then Theorem 1.3 holds for $G_{1}$ if and only if it holds for $G_{2}$.

Proof. Suppose Theorem 1.3 holds for $G_{2}$. Let $A_{1}, B_{1}$ be connected commuting $G_{1}$-cr subgroups of $G_{1}$. Then $A_{2}:=f\left(A_{1}\right)$ and $B_{2}:=f\left(B_{1}\right)$ are connected commuting $G_{2}$-cr subgroups of $G_{2}$ by Lemma 2.7(ii), so our hypothesis on $G_{2}$ implies that $A_{2} B_{2}$ is $G_{2}$-cr. Since $f\left(A_{1} B_{1}\right)=A_{2} B_{2}$, Lemma 2.7(ii) implies that $A_{1} B_{1}$ is $G_{1}$-cr.

Conversely, suppose Theorem 1.3 holds for $G_{1}$. Let $A_{2}, B_{2}$ be connected commuting $G_{2}$-cr subgroups of $G_{2}$. Then $A_{1}:=f^{-1}\left(A_{2}\right)^{0}$ and $B_{1}:=f^{-1}\left(B_{2}\right)^{0}$ are connected $G_{1}$-cr subgroups of $G_{1}$, by Lemma 2.7(ii). Since ker $f \subseteq Z\left(G_{1}\right)$ is linearly reductive, $A_{1}$ and $B_{1}$ satisfy the hypotheses of Lemma 3.11. Hence $A_{1} B_{1}$ is $G_{1}$-cr, by Lemma 3.11 and our hypothesis on $G_{1}$. Lemma 2.7(ii) now implies that $A_{2} B_{2}=f\left(A_{1} B_{1}\right)$ is $G_{2}$-cr.

Lemma 4.4. If Theorem 1.3 holds for each simple factor $G_{i}$ of $G$, then it holds for $G$.

Proof. Let $G, A$ and $B$ be as in the statement of Theorem 1.3. Multiplication gives an isogeny from $G_{1} \times \cdots \times G_{r} \times Z$ onto $G$, where $Z=Z(G)^{0}$. By Lemma 4.3, we may assume that $G=G_{1} \times \cdots \times G_{r} \times Z$. Let $\pi: G \rightarrow Z$ and $\pi_{i}: G \rightarrow G_{i}$ be the projection maps. Now $A$ (resp. $B, A B$ ) is $G$-cr if and only if $\pi_{i}(A)$ (resp. $\pi_{i}(B), \pi_{i}(A B)$ ) is $G_{i}$-cr for each $i$, by Lemma 2.7(i) (note that $\pi(A), \pi(B)$ and $\pi(A B)$ are automatically $Z$-cr as $Z$ is a torus). But $\pi_{i}(A B)=\pi_{i}(A) \pi_{i}(B)$ and $\left[\pi_{i}(A), \pi_{i}(B)\right]=\{1\}$, so Theorem 1.3 holds for $G$ if it holds for each $G_{i}$.

We do not have a uniform proof of Theorem 1.3, in fact we proceed by a series of case-bycase checks. First we consider the classical groups. 
4.1. Classical Groups. If $G$ is classical, then we obtain slightly stronger results (see Remark 4.7). First we consider the case $G=\mathrm{GL}(V)$. We believe that the following result is a standard fact in representation theory, but we have not been able to find a proof in the literature. The special case of Lemma 4.5 when $A$ and $B$ are connected reductive subgroups of $\mathrm{GL}(V)$ is proved in [10, Lem. 41] using facts from the representation theory of reductive groups. We are grateful to R. Tange for providing the argument given below.

Lemma 4.5. Let $V$ be a finite dimensional vector space over $k$. Suppose $A$ and $B$ are commuting subgroups of $\mathrm{GL}(V)$. Then $V$ is semisimple for the product $A B$ if and only if $V$ is semisimple for $A$ and $B$.

Proof. Suppose that $V$ is semisimple for $A$ and $B$. Let $C, D$, and $E$ be the $k$-subalgebras of $\operatorname{End}(V)$ spanned by $A, B$, and $A B$, respectively. Since $C$ and $D$ act faithfully and semisimply on $V, C$ and $D$ are semisimple $k$-algebras (cf. [6, Ch. XVII, Prop. 4.7]). Since $k$ is algebraically closed, and thus perfect, $C \otimes_{k} D$ is semisimple, by [4, $\S 7.6$ Cor. 4] (or [6, Ch. XVII, Thm. 6.4]). We have an epimorphism from $C \otimes_{k} D$ to $E$ given by $c \otimes d \mapsto c d$. It follows that $E$ is also semisimple (see [6, Ch. XVII, Prop. 2.2 and $\S 4]$ ), so $E$ acts semisimply on $V$ ([6, Ch. XVII, Prop. 4.7]). Thus $V$ is semisimple for $A B$, as required. The other implication follows from Clifford's Theorem.

Theorem 4.6. Theorem 1.3 holds for $G$ a simple group of classical type.

Proof. Let $A, B$ be commuting subgroups of $G$. Let $G_{1}$ be the classical group with the same Dynkin type as $G$ : so $G_{1}$ is either $\operatorname{SL}(V), \operatorname{Sp}(V)$ or $\operatorname{SO}(V)$. Let $\widetilde{G}$ be the simply connected cover of $G$. We have canonical projections $\widetilde{G} \rightarrow G$ and $\widetilde{G} \rightarrow G_{1}$. By Lemma 4.3, we can assume that $G=G_{1}$.

If $G=\mathrm{SL}(V)$, then $G$ is normal in $\operatorname{GL}(V)$, so the result follows from Proposition 2.8 and Lemma 4.5. The other two possibilities $G=\operatorname{Sp}(V)$ and $G=\operatorname{SO}(V)$ arise as the connected centralizer of an involution acting on $\operatorname{GL}(V)$. By hypothesis, $p \neq 2$, so the group of automorphisms generated by this involution is linearly reductive and Proposition 2.9 applies; cf. [1, Ex. 3.23]. Now $A$ and $B$ are $G$-cr if and only if $A$ and $B$ are GL $(V)$-cr, which happens if and only if $V$ is semisimple as an $A$ - and a $B$-module. By Lemma 4.5, this happens if and only if $V$ is semisimple for $A B$, which occurs if and only if $A B$ is $\mathrm{GL}(V)$-cr; by Proposition 2.9 again, this happens if and only if $A B$ is $G$-cr.

Remark 4.7. We did not need to assume that $A$ and $B$ were connected in Lemma 4.5. The proofs of Lemmas 4.3 and 4.4 and Theorem 4.6 also go through for non-connected $A$ and $B$, so Theorem 1.3 holds for non-connected $A$ and $B$ as well when all of the simple factors of $G$ are classical.

4.2. Exceptional Groups. Recall Theorem 2.15, Examination of the possible values for $N(X, G)$ in Table 1 shows that $N(X, G) \leq 7$ always. Thus, if $p>7$ (recall our convention that $p=\infty$ in characteristic zero!), Theorem 1.3 holds for simple exceptional groups simply because $A B$ is connected reductive. The remainder of this subsection is devoted to improving the bound on $p$; we show that $p>3$ will do.

We now prove Theorem 1.3 via a series of lemmas which exhaust all further possibilities. At various points we use inductive arguments involving Levi subgroups of groups of type $E_{6}$, $E_{7}$ and $E_{8}$; we are able to leave the $G_{2}$ and $F_{4}$ cases until last (Lemma 4.15) because groups of type $G_{2}$ and $F_{4}$ cannot arise as simple factors of these Levi subgroups. 
Lemma 4.8. Suppose $p>3$ and $X$ is a connected reductive group which has simple factors of rank at most 4. Suppose further that $X$ has no simple factor of type $A_{4}$ or $C_{4}$. Then, if $Y$ is a connected reductive subgroup of $X, Y$ is $X$-completely reducible.

Proof. Using Lemma 4.2, we reduce to the case when $X$ is simple. Thus we can list the possible types for $X: A_{1}, A_{2}, B_{2}, G_{2}, A_{3}, B_{3}, C_{3}, B_{4}, D_{4}, F_{4}$.

First suppose $X$ has one of the classical types in this list. Examining [7, Table 8.4], we see that all these types arise as subgroups of $F_{4}$ when $p>3$. By Table 1 , $N\left(Y, F_{4}\right) \leq 3<p$ for any connected reductive subgroup $Y$ of $F_{4}$. Since $X$ has classical type, we can invoke Theorem 2.16 to conclude that $Y$ is $X$-cr.

Finally, if $X$ has type $G_{2}$ or $F_{4}$, then $N(Y, X) \leq 3<p$, by Table 1, so $Y$ is $X$-cr, by a direct application of Theorem 2.15 .

Now we introduce some more notation to make the exposition easier. Given a $G$-completely reducible subgroup $A$ of the simple exceptional group $G$, let $H_{A}:=C_{G}(A)^{0}$. If $A$ and $B$ are connected, commuting $G$-cr subgroups of $G$, then to prove that $A B$ is $G$-cr, it suffices to show that $B$ is $H_{A}$-cr, by Propositions 2.12 and 3.9 (note that $C_{G}(A)$ need not be connected). The next lemma allows us to proceed through each remaining simple group in turn.

Lemma 4.9. Suppose $G$ is simple and Theorem 1.3 holds for all simple factors of all proper Levi subgroups of $G$. Then we may assume when proving Theorem 1.3 that $A \cap H_{A}$ is finite.

Proof. If $A \cap H_{A}$ is an infinite group, then $A \cap H_{A}$ contains a non-trivial (hence non-central) torus $S$ of $G$. In this case, $A B \subseteq A H_{A} \subseteq C_{G}(S)$, and $L:=C_{G}(S)$ is a proper Levi subgroup of $G$. By Corollary 2.10, $A$ and $B$ are commuting $L$-cr subgroups of $L$; thus, by Lemma 4.4 and the hypothesis, $A B$ is $L$-cr, and hence is $G$-cr by Corollary 2.10.

Lemma 4.10. Theorem 1.3 holds for $G$ simple of type $E_{6}$.

Proof. Let $A$ and $B$ be commuting $G$-cr subgroups of $G$. We show that $B$ is $H_{A^{-}}$-cr. Since any simple factor of a proper Levi subgroup of $G$ is of classical type, we may assume $A \cap H_{A}$ is finite, by Lemma 4.9 and Theorem 4.6. In particular, $A$ is not a non-trivial torus and $\operatorname{rk} A H_{A}=\operatorname{rk} A+\operatorname{rk} H_{A}$. If $\operatorname{rk} A H_{A}=\operatorname{rk} G$, then $A H_{A}$ is a regular reductive subgroup of $G$, and hence $H_{A}$ is a connected regular reductive subgroup of $G$. Since $p$ is good for $G$ and $B$ is $G$-cr, Proposition 2.11 shows that $B$ is $H_{A}$-cr, as required. Further, if $\mathrm{rk} B=\mathrm{rk} H_{A}$, then $B$ is regular in $H_{A}$ and hence $H_{A^{-}}$cr, by Lemma 2.5. Also, the result is trivial if $A$ or $B$ is trivial, so we assume this is not the case. We are therefore left to consider the cases where $1 \leq \operatorname{rk} B<\operatorname{rk} H_{A}<\operatorname{rk} A H_{A}<\operatorname{rk} G=6$. Thus rk $H_{A} \leq 4$.

Now Lemma 4.8 covers all these cases except $H_{A}=A_{4}$ or $C_{4}$. An examination of [7, Table 8.3] shows that if $H_{A}=A_{4}$, then $H_{A}$ is regular, so that $B$ is $H_{A^{-c r}}$ by Proposition 2.11, On the other hand, if $H_{A}=C_{4}$, then $A \subseteq C_{G}\left(H_{A}\right)^{0}=\{1\}$, a contradiction, which shows that this case cannot arise.

Lemma 4.11. Theorem 1.3 holds for $G$ simple of type $E_{7}$.

Proof. Any simple factor of a proper Levi subgroup of $G$ is either of classical type or of type $E_{6}$. By Theorem 4.6 and Lemmas 4.9 and 4.10, we may assume that $A \cap H_{A}$ is finite. Repeating the rank argument in the proof of Lemma 4.10, this means we only need to consider cases where $1 \leq \operatorname{rk} B<\operatorname{rk} H_{A}<\operatorname{rk} A H_{A}<\operatorname{rk} G=7$ and $A$ is not a torus. 
We now look at the possibilities for $H_{A}$ with $\mathrm{rk} H_{A} \leq 5$. For each one, we prove that $A B$ is $G$-cr, either directly or by proving that $B$ is $H_{A}$-cr. Again, Lemma 4.8 covers most of the cases; we are left to consider the possibility that

$$
H_{A}=A_{4}, C_{4}, A_{4} T_{1}, C_{4} T_{1}, A_{4} A_{1}, C_{4} A_{1}, A_{5}, B_{5}, C_{5}, D_{5},
$$

where $T_{1}$ denotes a 1-dimensional torus. We deal with these cases by examining [7, Table $8.2]$.

- If $H_{A}=A_{4}, A_{5}$ or $D_{5}$, then $H_{A}$ is regular, so $B$ is $H_{A^{-c r}}$ by Proposition 2.11,

- If $H_{A}=C_{4}$, then $A \subseteq C_{G}\left(H_{A}\right)=T_{1}$ is a torus. But this is impossible, because we assume $A$ is not a torus.

This also shows that the case $H_{A}=C_{4} A_{1}$ cannot arise, as there is no $A_{1}$ subgroup centralizing a $C_{4}$.

- If $H_{A}=A_{4} T_{1}$ or $C_{4} T_{1}$, then $A B \subseteq C_{G}\left(T_{1}\right)$, which is a proper Levi subgroup of $G$, so $A B$ is $G$-cr by Corollary 2.10, Lemma 4.4, Theorem 4.6 and Lemma 4.10.

- If $H_{A}=A_{4} A_{1}$, then since $\operatorname{rk} A+\operatorname{rk} H_{A}=\operatorname{rk} A H_{A}<\operatorname{rk} G=7$, we have $\operatorname{rk} A=1$. Then $A=A_{1}$ and, since $A \cap H_{A}$ is finite, there is a subgroup of type $A_{1} A_{1}$ in $C_{G}\left(A_{4}\right)^{0}$. But $C_{G}\left(A_{4}\right)^{0}=A_{2} T_{1}$, which does not contain an $A_{1} A_{1}$ subgroup. Thus this case cannot arise.

- If $H_{A}=B_{5}$, then $H_{A} \subset D_{6}$ (cf. Remark 2.17), which is regular in $G$. Moreover, the $B_{5}$ is the connected centralizer of the graph automorphism of order 2 for $D_{6}$. Now $B$ is $D_{6}$-cr, by Proposition 2.11 (note that $p>2$ ), and hence $H_{A^{-}}$,r, by Proposition 2.9 .

- There is no subgroup of type $C_{5}$ in $E_{7}$.

This completes the proof of the lemma.

Now we need to deal with the case when $G$ has type $E_{8}$. This is more involved because we actually allow $p$ to be 5 , which is a bad prime for $E_{8}$.

Lemma 4.12. Suppose $p>3$. Let $G$ be simple of type $E_{8}$ and let $H$ be a connected reductive regular subgroup of $G$ such that $H$ is not simple of rank 8. Then for any subgroup $K$ of $H$, $K$ is $G$-completely reducible if and only if $K$ is $H$-completely reducible.

Proof. Let $H_{1}, \ldots, H_{r}$ be the simple factors of $H$ and let $K_{i}$ be the projection of $K$ to each $H_{i}$. By Lemma 4.2, it is enough to prove the result for each $i$ with $H$ replaced by $H_{i}$ and $K$ replaced by $K_{i}$. Now $H_{i}$ has semisimple rank at most 7 by hypothesis, so $H_{i}$ is a regular subgroup of a proper Levi subgroup $L$ of $G$. Since $p>3$ and $L$ has no simple factors of type $E_{8}, p$ is good for $L$, so the required result follows from Proposition 2.11 and Corollary 2.10.

Lemma 4.13. Let $G$ be simple of type $E_{8}$ and let $X$ be a simple subgroup of $G$ such that $\operatorname{rk} X \leq 6, X$ is not of type $C_{4}$ and $X$ is not a non-regular subgroup of type $A_{4}$. Then for any connected reductive subgroup $Y$ of $X$, if $Y$ is $G$-completely reducible, then $Y$ is $X$-completely reducible.

Proof. By Lemma 4.8, we need only consider the cases when $X$ is regular and of type $A_{4}$ or $X$ has rank either 5 or 6 . We deal with these cases by examining [7, Table 8.1]. Let $Y$ be a $G$-cr subgroup of $X$. 
- If $X=B_{5}$, then $X \subset D_{6}$ (cf. Remark 2.17), which is regular in $G$. Moreover, the $B_{5}$ subgroup of $D_{6}$ arises as the connected centralizer of an involution of $D_{6}$. By Lemma 4.12, $Y$ is $D_{6}$-cr, so by Proposition [2.9, $Y$ is $X$-cr (note that $p>2$ ). The same argument works for $B_{6} \subset D_{7} \subset G$, where $D_{7}$ in $G$ is regular.

- If $X=A_{5}, A_{6}, D_{5}, D_{6}$ or $E_{6}$, then $X$ is regular in $G$, so we are done by Lemma 4.12 , If $X=A_{4}$, then $X$ is regular in $G$ by hypothesis, so the same argument holds.

- There is no subgroup of type $C_{5}$ or $C_{6}$ in $E_{8}$.

This completes the proof of the lemma.

Lemma 4.14. Theorem 1.3 holds for $G$ simple of type $E_{8}$.

Proof. Any simple factor of a proper Levi subgroup of $G$ is either of classical type or of type $E_{6}$ or $E_{7}$. By Theorem 4.6 and Lemmas 4.9, 4.10 and 4.11, we may assume that $A \cap H_{A}$ is finite. We can assume that $A$ and $H_{A}$ have rank at least 1 . Hence if $A H_{A}$ is regular in $G$, then, since $B$ is $G$-cr, Lemma 4.12 implies that $B$ is $A H_{A^{-}}$cr, so $B$ is $H_{A^{-}}$cr by Proposition 2.8. Thus we may assume that $\operatorname{rk} A H_{A}<\operatorname{rk} G$. Repeating the rank argument in the proof of Lemma 4.10, this means we only need to consider cases where $1 \leq \operatorname{rk} B<\operatorname{rk} H_{A}<\operatorname{rk} A H_{A}<\operatorname{rk} G=8$ and $A$ is not a torus.

We now look at the possibilities for $H_{A}$ with $\mathrm{rk} H_{A} \leq 6$. For each one, we prove that $A B$ is $G$-cr, either directly or by proving that $B$ is $H_{A^{-}}$cr. We use [7, Table 8.1] to deal with the various cases.

- Suppose $H_{A}$ is not semisimple: say $S$ is a non-trivial central torus in $H_{A}$. Then $A B$ is contained in $C_{G}(S)$, a proper Levi subgroup of $G$, so $A B$ is $G$-cr by Corollary 2.10, Lemma 4.4, Theorem 4.6, Lemma 4.10 and Lemma 4.11.

- Suppose $H_{A}$ has a non-regular $A_{4}$-factor. Then $C_{G}\left(H_{A}\right) \subseteq C_{G}\left(A_{4}\right)$ is trivial; but this is impossible, because $A$ is non-trivial.

- Suppose $H_{A}=C_{4}$. There are two cases. First, suppose $H_{A}$ is contained in a regular $E_{6}$. The connected centralizers of $H_{A}$ and the $E_{6}$ are the same: this centralizer is of type $A_{2}$. Thus $A H_{A}$ is contained in $M:=E_{6} A_{2}$, which has rank 8 and hence is regular in $G$. By Lemma 4.12, $A$ and $B$ are $M$-cr, so $A B$ is $M$-cr by Lemma 4.4, Theorem 4.6 and Lemma 4.10, Hence $A B$ is $G$-cr by Lemma 4.12.

Second, suppose $H_{A}$ is contained in a regular $A_{7}$. Then $H_{A}$ is the connected centralizer of an involution of the $A_{7}$ (cf. Remark 2.17), so Lemma 4.12 and Proposition 2.9 imply that $B$ is $H_{A}$-cr.

- Suppose $H_{A}=C_{4} H$, where $H$ is connected and reductive but not a torus. Then $H$ is a subgroup of $C_{G}\left(C_{4}\right)^{0}=A_{2}$ and $H$ is not a torus, so $C_{G}\left(H_{A}\right)^{0}=C_{A_{2}}(H)^{0}$ is a torus. But $A$ is a subgroup of $C_{G}\left(H_{A}\right)^{0}$ of semisimple rank at least one, a contradiction.

- Suppose $H_{A}$ is simple and not of type $A_{4}$ or type $C_{4}$. Since $H_{A}$ has rank at most 6 , Lemma 4.13 implies that $B$ is $H_{A^{-}}$-cr.

- Suppose every simple factor of $H_{A}$ has rank at most 4 and $H_{A}$ has no simple factors of type $A_{4}$ or $C_{4}$. Lemma 4.8 implies that $B$ is $H_{A^{-}}$cr.

- Otherwise, write $H_{A}=X_{1} X_{2}$, where $X_{2}$ is semisimple and has every simple factor of rank at most 3 , and $X_{1}$ is semisimple and has every simple factor of rank at least 4 . Then $X_{1}$ is simple, and we may assume that $X_{1} \neq C_{4}$ and $X_{1}$ is not a non-regular $A_{4}$. Note that $X_{1} \neq C_{5}, C_{6}$ since $G$ does not contain a $C_{5}$ or a $C_{6}$. Suppose first that $X_{1}$ is regular and of type $A_{4}$, or is of type $A_{5}$ or $D_{5}$. Then $A B$ is a subset of 
$X_{1} C_{G}\left(X_{1}\right)^{0}$, which is of type $A_{4} A_{4}, A_{5} A_{1} A_{2}$ or $D_{5} A_{3}$ respectively. Thus $X_{1} C_{G}\left(X_{1}\right)^{0}$ is regular in $G$. It now follows from Lemma 4.4. Theorem 4.6 and Lemma 4.12 that $A B$ is $G$-cr (compare the first $H_{A}=C_{4}$ case above).

Now suppose that $X_{1}$ is of type $A_{6}, B_{6}, D_{6}$ or $E_{6}$. Then $H_{A}=X_{1}$ since $H_{A}$ has rank at most 6 , so $B$ is $H_{A^{-}}$-cr by Lemma 4.13,

Finally, suppose that $X_{1}$ is of type $B_{5}$. If $H_{A}=B_{5}$ then $B$ is $H_{A^{-}}$cr by Lemma4.13, Otherwise we must have $H_{A}=B_{5} A_{1}$, since $H_{A}$ has rank at most 6 . Our hypotheses on the rank of $A H_{A}$ now imply that $A=A_{1}$, so the $B_{5}$-factor is centralized by an $A_{1} A_{1}$. Now $B_{5}$ sits inside a subgroup of $G$ of type $D_{6}$, and this $D_{6}$ has a group of type $A_{1} A_{1}$ as its connected centralizer. Thus we have two groups of type $A_{1} A_{1}$ inside $C_{G}\left(B_{5}\right)^{0}=B_{2}$. As $p>2$, these two subgroups must be $B_{2}$-conjugate. Hence $A B$ is contained in $D_{6} A_{1} A_{1}$, which is regular. It now follows from Lemma 4.4, Theorem 4.6 and Lemma 4.12 that $A B$ is $G$-cr (compare the first $H_{A}=C_{4}$ case above).

This exhausts all the possibilities, so the lemma is proved.

Lemma 4.15. Theorem 1.3 holds for $G$ simple of type $G_{2}$ or $F_{4}$.

Proof. Suppose $G$ is simple of type $G_{2}$. Then $G$ arises as the connected centralizer of $D_{4}$ under the triality graph automorphism. Since $p>3$, this automorphism generates a linearly reductive group. Thus a subgroup of $G$ is $G$-cr if and only if it is $D_{4}$-cr, by Proposition 2.9. But Theorem 1.3 holds for $D_{4}$ by Theorem [4.6, so it holds for $G$.

Now suppose $G$ is simple of type $F_{4}$. In this case $G$ arises as the connected centralizer of an involution of $E_{6}$. Since $p>2$, this automorphism generates a linearly reductive group. Thus a subgroup of $G$ is $G$-cr if and only if it is $E_{6}$-cr, by Proposition 2.9, But Theorem 1.3 holds for $E_{6}$ by Lemma 4.10, so it holds for $G$.

Theorem 1.3 now follows from Lemma 4.4, together with Theorem 4.6 and Lemmas 4.10, 4.11, 4.14 and 4.15.

Combining Theorems 1.1 and 1.3 yields the following.

Corollary 4.16. Suppose that $G$ is connected and that $p$ is good for $G$ or $p>3$. If $A$ and $B$ are commuting connected reductive subgroups of $G$, then $A B$ is $G$-completely reducible if and only if $A$ and $B$ are $G$-completely reducible.

We provide a reformulation of Theorem 1.3 which shows more clearly its relation to Theorem 1.1.

Corollary 4.17. Suppose that $G$ is connected and that $p$ is good for $G$ or $p>3$. Suppose $N \subseteq H$ are connected subgroups of $G$ such that $N$ is normal in $H$. If there exists a connected $G$-completely reducible subgroup $M$ of $C_{G}(N)$ such that $H=M N$, then $H$ is $G$-completely reducible if and only if $N$ is $G$-completely reducible.

Proof. If $H$ is $G$-cr, then $N$ is $G$-cr by Theorem 1.1. On the other hand, if $N$ is $G$-cr, then Theorem 1.3 applies, with $A=N, B=M$ and $A B=H$.

Remark 4.18. One consequence of Theorem 1.3 is that the converse of Corollary 3.10 is true for connected groups if $p$ is good or $p>3$; this follows from Proposition 3.9, However, we give examples in Section 5 which show that the converse to Corollary 3.10, and hence Theorem 1.3, is false without the restriction on the characteristic. 
Remark 4.19. By Lemma 3.11, Theorem 1.3 holds under the weaker hypotheses that $A$ and $B$ are $G$-cr, $A$ normalizes $B,[A, B]$ is linearly reductive and $[A, B]$ centralizes $A$.

\section{Counterexamples And Extensions}

As promised in Remark 4.18, in this section we provide examples which show that Theorem 1.3 fails in general without the hypotheses of connectedness and good characteristic. We also give an extension to Theorem 1.3 which shows that one can remove the connectedness assumption at least in some cases. Our first example shows that, even in good characteristic, Theorem 1.3 fails for disconnected groups.

Example 5.1. Suppose $p=2$ and $m \geq 4$ is even. Define $\phi \in \operatorname{Aut}\left(\mathrm{GL}_{2 m}\right)$ by $\phi(g)=$ $J\left(g^{t}\right)^{-1} J^{-1}$, where $g^{t}$ denotes the matrix transpose of $g, J=\left(\begin{array}{cc}0 & I_{m} \\ I_{m} & 0\end{array}\right)$ and $I_{m}$ is the $m \times m$ identity matrix. Set $A=\langle\phi\rangle$ and $G=A \ltimes \mathrm{GL}_{2 m}$. Let $B=\mathrm{Sp}_{m}$ embedded diagonally in the maximal rank subgroup $\mathrm{Sp}_{m} \times \mathrm{Sp}_{m}$ of $\mathrm{Sp}_{2 m}$, and consider the canonical embedding of $\mathrm{Sp}_{2 m}$ in $G^{0}$. We can identify $\operatorname{Sp}_{2 m}$ with $C_{G}(A)^{0}$. By [1, Ex. 3.45], $B$ is $G^{0}$-cr but not $\mathrm{Sp}_{2 m}$-cr.

Observe that $\mathrm{Sp}_{2 m}$ is $G^{0}$-ir. Thus if $\lambda \in Y\left(G^{0}\right)=Y(G)$ with $A \mathrm{Sp}_{2 m} \subseteq P_{\lambda}$, then $\lambda$ belongs to $Y\left(Z\left(G^{0}\right)\right)$ and $R_{u}\left(P_{\lambda}\right)=\{1\}$, so $A \operatorname{Sp}_{2 m} \subseteq P_{\lambda}=L_{\lambda}$. This shows that $A \mathrm{Sp}_{2 m}$ is $G$-cr. Thus the normal subgroup $A$ of $A \mathrm{Sp}_{2 m}$ is $G$-cr (Theorem 1.1). By Proposition 2.12, $B$ is $G$-cr. However, $B$ is not $\mathrm{Sp}_{2 m}$-cr, so Proposition 2.12 implies that $B$ is not $A \mathrm{Sp}_{2 m}$-cr. We have $A \mathrm{Sp}_{2 m}=C_{G}(A)$, so $B$ is not $C_{G}(A)$-cr.

Thus we have commuting subgroups $A$ and $B$ which are $G$-cr, but such that $B$ is not $C_{G}(A)$-cr. Hence, by Proposition 3.9, $A B$ is not $G$-cr. In particular, even though $p=2$ is good for $G^{0}=\mathrm{GL}_{2 m}$, Theorem 1.3 and the converse to Corollary 3.10 fail for these subgroups of the disconnected group $G$.

Remark 5.2. We cannot have an example of this kind inside a connected group: for a nontrivial unipotent subgroup $A$ can never be $G$-cr if $G$ is connected (see Remark 2.3).

The following example, due to M. Liebeck, shows that Theorem 1.3, and hence the converse to Corollary [3.10, can also fail for connected groups in bad characteristic.

Example 5.3. Suppose $p=2$. We show that there exist connected commuting subgroups $A, B$ of $\mathrm{Sp}_{8}$ such that $A, B$ are $\mathrm{Sp}_{8}$-cr but $A B$ is not.

Let $A=B=\mathrm{SL}_{2}$ and let $V_{A}, V_{B}$ be the natural modules for $A, B$ respectively. Choose symplectic forms $(\cdot, \cdot)_{A},(\cdot, \cdot)_{B}$ for $V_{A}, V_{B}$ respectively. Then $\operatorname{SL}\left(V_{A}\right)=\operatorname{Sp}\left(V_{A}\right)$ and $\operatorname{SL}\left(V_{B}\right)=$ $\operatorname{Sp}\left(V_{B}\right)$. Set $W:=V_{A} \otimes V_{B}$ with the symplectic form $(\cdot, \cdot)_{W}$ given by

$$
\left(u_{1} \otimes u_{2}, v_{1} \otimes v_{2}\right)_{W}:=\left(u_{1}, v_{1}\right)_{A}\left(u_{2}, v_{2}\right)_{B} .
$$

Then $A, B$ and $A \times B$ act on $W$ in the obvious way, and these actions preserve $(\cdot, \cdot)_{W}$. Below we shall be interested in $A$-stable subspaces of $W$. For $v \in V_{B}$, set

$$
V_{A} \otimes v:=\left\{u \otimes v \mid u \in V_{A}\right\},
$$

a subspace of $W$. Since $W$ is the $A$-module direct sum of the irreducible $A$-modules $V_{A} \otimes v_{1}$ and $V_{A} \otimes v_{2}$, where $v_{1}$ and $v_{2}$ are any two linearly independent vectors in $V_{B}$, we see that $A$ acts completely reducibly on $W$ and the proper non-trivial $A$-stable subspaces of $W$ are 
precisely the subspaces of the form $V_{A} \otimes v$ for some $v \in V_{B}$. In particular, the $A$-stable subspaces of $W$ have dimension 0,2 or 4 .

There exists an $A$-module isomorphism $\phi_{A}: V_{A} \rightarrow V_{A}^{*}$ corresponding to the symplectic form on $V_{A}$. Define $\phi_{B}: V_{B} \rightarrow V_{B}^{*}$ analogously and identify $W^{*}$ with $V_{A}^{*} \otimes V_{B}^{*}$ via the $(A \times B)$-module isomorphism $\psi:=\phi_{A} \otimes \phi_{B}$; this is precisely the isomorphism corresponding to the symplectic form on $W$, and gives rise to a symplectic form $(\cdot, \cdot)_{W^{*}}$ on $W^{*}$ given by

$$
\left(\psi\left(w_{1}\right), \psi\left(w_{2}\right)\right)_{W^{*}}:=\left(w_{1}, w_{2}\right)_{W} .
$$

Consider the $(A \times B)$-module $U:=W \oplus W^{*}$ endowed with the direct sum symplectic form, which we denote by $(\cdot, \cdot)_{U}$. The $(A \times B)$-action preserves the symplectic structure, so we can regard $A, B$ and $A \times B$ as subgroups of $\operatorname{Sp}(U)$. If $M$ is an $A$-stable subspace of $U$ then we have a short exact sequence of $A$-modules

$$
\{0\} \rightarrow M \cap W \rightarrow M \rightarrow \pi_{2}(M) \rightarrow\{0\},
$$

where $\pi_{2}: U \rightarrow W^{*}$ is the canonical projection. Since the second and fourth term have even dimension, $M$ must have even dimension. In particular, if $M$ is isotropic then $M$ has dimension 0, 2 or 4 . We claim that if $M$ is any 4-dimensional $A$-stable isotropic subspace of $U$, then there exists an $A$-stable isotropic complementary subspace. To establish this, we observe that such a subspace $M$ must either be of the form $V_{A} \otimes v \oplus V_{A}^{*} \otimes f$ for some $0 \neq v \in V_{A}$ and $0 \neq f \in V_{A}^{*}$, or of the form $M_{\theta}:=\operatorname{graph}(\theta)$, where $\theta: W \rightarrow W^{*}$ is an $A$-module isomorphism (note that $W$ and $W^{*}$ are not isotropic). Any subspace of the first type is isotropic, so we can take a complement to be $V_{A} \otimes v^{\prime} \oplus V_{A}^{*} \otimes f^{\prime}$, where $v$ and $v^{\prime}$ (resp. $f$ and $f^{\prime}$ ) are linearly independent in $V_{A}$ (resp. $V_{A}^{*}$ ). Given $M$ of the form $M_{\theta}$, choose any $0 \neq v \in V_{B}$. The $A$-stable subspace $\theta\left(V_{A} \otimes v\right)$ of $W^{*}$ is of the form $V_{A}^{*} \otimes f$ for some $f \in V_{B}^{*}$. Choose $g \in V_{B}^{*}$ such that $f$ and $g$ are linearly independent; then $M_{\theta}$ and $V_{A} \otimes v \oplus V_{A}^{*} \otimes g$ intersect trivially, and the latter subspace is isotropic. This proves the claim.

We now prove that $A$ is $\operatorname{Sp}(U)$-cr; the analogous result for $B$ follows by symmetry. The parabolic subgroups of $\operatorname{Sp}(U)$ are precisely the stabilizers of flags

$$
\mathcal{F}:\{0\} \subset M_{1} \subset M_{2} \subset \cdots \subset M_{r} \subset U
$$

of isotropic subspaces of $U$. Moreover, a parabolic subgroup $P$ is opposite to the stabilizer of $\mathcal{F}$ if and only if $P$ is the stabilizer of a flag $\{0\} \subset N_{1} \subset N_{2} \subset \cdots \subset N_{r} \subset U$ of isotropic subspaces such that $U=M_{i}^{\perp} \oplus N_{i}$ for each $i$. (Both of these facts can easily be established by considering the parabolic subgroups containing the standard maximal torus $S$ described in [3, Ch. $\mathrm{V}, \S 23.3]$.) Let $\mathcal{F}$ be a flag of $A$-stable isotropic subspaces of $U$. There are only three possible types of flag to check. If $\mathcal{F}$ has the form $\{0\} \subset M \subset U$ with $\operatorname{dim} M=4$, then we are done, by the previous claim (note that $M=M^{\perp}$ ). Now suppose that $\mathcal{F}$ has the form $\{0\} \subset M_{1} \subset M_{2} \subset U$, where $\operatorname{dim} M_{1}=2$ and $\operatorname{dim} M_{2}=4$. By the previous claim, there exists a 4-dimensional $A$-stable isotropic subspace $N_{2}$ of $U$ such that $U=M_{2}^{\perp} \oplus N_{2}$. Since $A$ acts completely reducibly on $U$, there exists an $A$-stable complement $N_{1}$ to $M_{1}^{\perp} \cap N_{2}$ in $N_{2}$. Then $N_{1}$ is an isotropic $A$-stable complement to $M_{1}^{\perp}$ and $N_{1} \subset N_{2}$, as required. Finally, suppose that $\mathcal{F}$ has the form $\{0\} \subset M \subset U$ with $\operatorname{dim} M=2$. It is easy to show, by listing the possibilities for $M$ as in the previous claim, that $M$ is contained in an $A$-stable 4-dimensional isotropic subspace of $U$, and we can use the argument of the second case to prove that $M^{\perp}$ has an $A$-stable isotropic complement in $U$. This completes the proof that $A$ is $\operatorname{Sp}(U)$-cr. 
We finish by proving that the isotropic $(A \times B)$-stable subspace $M_{\psi}=\{(w, \psi(w)) \mid w \in W\}$ does not admit an isotropic $(A \times B)$-stable complement, which proves that $A \times B$ is not $\operatorname{Sp}(U)$-cr; we repeat the argument of [1, Ex. 3.45] for the convenience of the reader. Suppose that $N$ is such a complement. It follows from the discussion above on $A$-stable subspaces of $U$ that $N$ must be of the form $M_{\theta}$ for some $(A \times B)$-module isomorphism $\theta: W \rightarrow W^{*}$. Since $A \times B$ acts irreducibly on $W$ and $W^{*}$, we must have $\theta=a \psi$ for some $a \in k$, by Schur's Lemma. For $u, v \in W$, we have

$$
\begin{aligned}
((u, \theta(u)),(v, \theta(v)))_{U} & =((u, a \psi(u)),(v, a \psi(v)))_{U} \\
& =(u, v)_{W}+a^{2}(\psi(u), \psi(v))_{W^{*}} \\
& =\left(1+a^{2}\right)(u, v)_{W} .
\end{aligned}
$$

As $N$ is isotropic, this expression is identically 0 , so we must have $a=1$. But then $N=M$, a contradiction.

Remark 5.4. We can endow each of $V_{A}$ and $V_{B}$ (and hence the spaces $V_{A}^{*}, V_{B}^{*}, W, W^{*}$ and $U$ ) in Example 5.3 with quadratic forms compatible with the given symplectic forms. The actions of $A, B$ and $A \times B$ on the various spaces are compatible with these quadratic forms, so we can regard $A, B$ and $A \times B$ as subgroups of $\mathrm{SO}(W), \mathrm{SO}(U)$, etc.; for example, the image of $A \times B$ in $\mathrm{Sp}(W)$ is precisely $\mathrm{SO}(W)$. Parabolic subgroups of $\mathrm{SO}(U)$ correspond to stabilizers of flags of totally singular subspaces of $U$. An argument similar to that of Example 5.3 shows that $A$ and $B$ are $\mathrm{SO}(U)$-cr but $A \times B$ is not.

We can use this result to provide counterexamples for exceptional groups as well. For example, the group $\mathrm{SO}_{8}(k)$ has type $D_{4}$ and the exceptional group of type $E_{6}$ has a Levi subgroup of type $D_{4}$. Since $G$-complete reducibility is invariant under taking isogenies, we can view $A, B$ and $A \times B$ as subgroups of $E_{6}$ in this way. The subgroups $A$ and $B$ are $D_{4}$-cr, hence they are $E_{6}$-cr, by Proposition 2.8 and Corollary 2.10, however, the product $A B$ is not $D_{4}$-cr, so cannot be $E_{6}$-cr, again by Proposition 2.8 and Corollary 2.10.

Example 5.5. We can modify Example 5.3 to obtain a similar counterexample involving finite subgroups rather than connected ones. Take $k$ to be the algebraic closure of the field with two elements. We replace $A$ (resp. $B$ ) with the finite subgroup $A(q)$ (resp. $B(q)$ ), where $q$ is a power of 2 ; for $q$ sufficiently large, [1, Lem. 2.10] implies that $A(q)$ and $B(q)$ are $\mathrm{Sp}_{8}$-cr but $A(q) B(q)$ is not.

Examples 5.3 and 5.5 show that even if $A, B$ and $A^{0} B^{0}$ are $G$-cr, $A B$ need not be $G$-cr: passing to finite extensions does not preserve $G$-complete reducibility. We can, however, identify one special case in which this works.

Proposition 5.6. Let $A, B$ be $G$-completely reducible subgroups of $G$ such that $A$ normalizes $B, A^{0}$ centralizes $B^{0}$ and the index of $(A B)^{0}$ in $A B$ is coprime to $p$. Then $A B$ is $G$-completely reducible if and only if $(A B)^{0}$ is $G$-completely reducible. In particular, $A B$ is $G$-completely reducible if $p$ is good for $G^{0}$.

Proof. Since $A B /(A B)^{0}$ is linearly reductive, the first assertion follows from Corollary 3.7(ii). Clearly, $(A B)^{0}=A^{0} B^{0}$. Since $A^{0}, B^{0}$ are $G^{0}$-cr by Theorem 1.1 and Proposition 2.12, the second assertion follows from Theorem 1.3 and Proposition 2.12 ,

Note that Proposition 5.6 is consistent with Example [5.1, for in that case $p=2$ and the index of $(A B)^{0}$ in $A B$ is also 2 , whence $A B /(A B)^{0}$ is not linearly reductive. 
In our final examples we return to the case that $N$ is a normal subgroup of $H$ and $M$ is a subgroup of $H$ such that $H=M N$ (cf. Section 3). We show that even if $H$ is $G$-cr and $M$ is a complement to $N, M$ need not be $G$-cr (see the discussion following Question 1.2).

Example 5.7. Let $p=2$, let $H$ be the symmetric group $S_{3}$ embedded irreducibly in $G=\mathrm{GL}_{2}$ and let $N$ be the subgroup of $H$ of order 3 . Any subgroup of $H$ of order 2 is a complement to $N$, but such a subgroup, being unipotent, cannot be $G$-completely reducible (Remark 2.3), since $G$ is connected.

Example 5.8. Suppose $p=2$ and $m \geq 4$ is even. Let $H$ be the maximal rank subgroup $\mathrm{Sp}_{m} \times \mathrm{Sp}_{m}$ of $G:=\mathrm{Sp}_{2 m}$, let $N=\{1\} \times \mathrm{Sp}_{m}, M^{\prime}=\mathrm{Sp}_{m} \times\{1\}$ and let $M$ be $\mathrm{Sp}_{m}$ embedded diagonally in $H$. Then $H, N$ and $M^{\prime}$ are $G$-cr, $M$ and $M^{\prime}$ are both complements to $N$ in $H$, but $M$ is not $G$-cr (see [1, Ex. 3.45]). It is easy to check that $H=N_{G}(N)^{0}$, and it follows from Proposition 2.12 and Lemma $2.7(\mathrm{i})$ that $M$ is $N_{G}(N)$-cr. This shows that Corollary 3.10 is false if we replace $C_{G}(N)$ with $N_{G}(N)$.

Acknowledgements: We would like to thank M.W. Liebeck, C.W. Parker, G.R. Robinson, and R. Tange for helpful discussions. We are also grateful to the referees for their helpful comments.

The authors acknowledge the financial support of EPSRC Grant EP/C542150/1 and Marsden Grant UOC0501. Part of the research for this paper was carried out while the authors were staying at the Mathematical Research Institute Oberwolfach supported by the "Research in Pairs" programme. Also part of this research was carried out during a visit by the second author to the University of Southampton: he is grateful to the members of the School of Mathematics for their hospitality.

\section{REFERENCES}

[1] M. Bate, B. Martin, G. Röhrle, A Geometric Approach to Complete Reducibility, Invent. Math. 161, no. 1, (2005), 177-218.

[2] M. Bate, B. Martin, G. Röhrle, R. Tange, Complete Reducibility and Separability, preprint (2007), http://arxiv.org/abs/0709.3803v3.

[3] A. Borel, Linear Algebraic Groups, Graduate Texts in Mathematics, 126, Springer-Verlag 1991.

[4] N. Bourbaki, Algèbre, Chapitre 8, Modules et Anneaux Semi-Simples, Hermann, Paris 1958.

[5] J.C. Jantzen, Nilpotent Orbits in Representation Theory, in Lie Theory. Lie Algebras and Representations. Progress in Math. vol. 228, J-P. Anker, B. Orsted, eds. Birkhäuser Boston, 2004.

[6] S. Lang, Algebra, 3rd ed. Addison-Wesley, Reading, Massachusetts, 1993.

[7] M.W. Liebeck, G.M. Seitz, Reductive subgroups of exceptional algebraic groups. Mem. Amer. Math. Soc. no. $\mathbf{5 8 0}$ (1996).

[8] B. Martin, Reductive subgroups of reductive groups in nonzero characteristic, J. Algebra 262 (2003), no. 2, 265-286.

[9] _ A normal subgroup of a strongly reductive subgroup is strongly reductive, J. Algebra 265 (2003), no. $2,669-674$.

[10] G. McNinch, D. Testerman, Completely Reducible SL(2)-homomorphisms, Trans. Amer. Math. Soc. 359 (2007), 4489-4510.

[11] M. Nagata, Complete reducibility of rational representations of a matric group, J. Math. Kyoto University 1 (1961), 87-99.

[12] R.W. Richardson, On orbits of algebraic groups and Lie groups, Bull. Austral. Math. Soc. 25 (1982), no. $1,1-28$. 
[13] J-P. Serre, The notion of complete reducibility in group theory, Moursund Lectures, Part II, University of Oregon, 1998, http://arxiv.org/abs/math/0305257v1.

[14] _ Complète Réductibilité, Séminaire Bourbaki, 56ème année, 2003-2004, nº 932.

[15] T.A. Springer, R. Steinberg, Conjugacy classes, Seminar on algebraic groups and related finite groups, Lecture Notes in Mathematics, 131, Springer-Verlag, Heidelberg (1970), 167-266.

Christ Church College, Oxford University, Oxford, OX1 1DP, UK

E-mail address: bate@maths.ox.ac.uk

Mathematics and Statistics Department, University of Canterbury, Private Bag 4800, Christchurch 1, New Zealand

E-mail address: B.Martin@math. canterbury.ac.nz

Fakultät für Mathematik, Ruhr-Universität Bochum, D-44780 Bochum, Germany

E-mail address: gerhard.roehrle@rub.de 